Keywords:

Flux tower

Phytosociology

Serra do Mar State Park

Histórico:

Recebido 04/10/2016

Aceito 25/I I/2016

Palavras chave:

Torre de fluxo

Fitossociologia

Parque Estadual da Serra do Mar

${ }^{+}$Correspondência: nidiamarchiori@yahoo.com.br

DOI:
Nidia Mara Marchiori ${ }^{1+}$, Humberto Ribeiro Rocha², Jorge Yoshio Tamashiro', Marcos Pereira Marinho Aidar ${ }^{3}$

\section{TREE COMMUNITY COMPOSITION AND ABOVEGROUND BIOMASS IN A SECONDARY ATLANTIC FOREST, SERRA DO MAR STATE PARK, SÃO PAULO, BRAZIL}

ABSTRAT: Projects involving floristic-phytosociological surveys are becoming increasingly frequent and is a very important tool to access the biodiversity, status of succession and biomass and carbon storage, guiding conservation and management strategies. These studies are particularly important in Atlantic Forest, which is considered a hotspot in terms of biodiversity, endemism and impacts. São Paulo State lost more than $80 \%$ of original forest and, nowadays, remains only isolated patches with a variety stage of succession and history of use. The aim of this study was to characterize the structure, composition and biomass of the woody plant community in a Montane Ombrophilous Dense Forest, Serra do Mar State Park. All trees with DBH $\geq 4.8 \mathrm{~cm}$ were sampled in I ha plot area, totaling I,704 individuals belonging to 38 botanical families and 143 species. The highest species richness was found in the Myrtaceae and Lauraceae families, and the greatest value of abundance and Importance (IV) was observed in the Arecaceae and Euphorbiaceae. The Shannon index $\left(\mathrm{H}^{\prime}\right)$ was 3.7 nats.ind. ${ }^{-1}$ and the Pielou's evenness index $(J) 0.7$, characterizing a very diverse community with heterogeneous distribution of individuals by species. The aboveground biomass was $166.3 \mathrm{Mg} \cdot \mathrm{ha}^{-1}$, similar to others studies in Atlantic forests. The forest composition, biomass and the history of land use indicate a middle secondary stage of regeneration, but evolving to a more mature condition.

\section{COMPOSIÇÃO DA COMUNIDADE ARBÓREA E BIOMASSA AÉREA EM UMA FLORESTA ATLÂNTICA SECUNDÁRIA, PARQUE ESTADUAL DA SERRA DO MAR, SÃO PAULO, BRAZIL}

RESUMO: Trabalhos envolvendo levantamentos florístico-fitossociológicos são cada vez mais frequentes e são uma ferramenta importante para acessar a biodiversidade, estágio de sucessão e acúmulo de biomassa e carbono, norteando estratégias de conservação e manejo. Esses estudos são particularmente importantes para a Mata Atlântica, considerada um hotspot em função da biodiversidade, endemismo e impactos. $O$ Estado de São Paulo perdeu mais de $80 \%$ de sua floresta original e, atualmente, existem somente fragmentos isolados, com diferentes estágios de sucessão e histórico de uso. O objetivo desse estudo foi caracterizar a vegetação, a estrutura e a biomassa de um componente arbóreo localizado em Floresta Ombrófila Densa Montana, Parque Estadual da Serra do Mar. Foram amostrados indivíduos com DAP $\geq 4,8 \mathrm{~cm}$ em I ha, totalizando I.704 indivíduos pertencentes a 38 famílias botânicas e 143 espécies. A maior riqueza de espécies foi encontrada nas famílias Myrtaceae e Lauraceae e a maior abundância e Valor de Importância (VI) em Arecaceae e Euphorbiaceae. O índice de Shannon ( $\left.\mathrm{H}^{\prime}\right)$ foi de 3,7 nats.ind. ${ }^{-1}$ e a equabilidade de Pielou (J) 0,7, indicando que a comunidade da área é bastante diversa, contudo a distribuição dos indivíduos pelas espécies não é homogênea. A biomassa aérea foi de $166,3 \mathrm{Mg} \cdot \mathrm{ha}^{-1}$, semelhante ao encontrado em outros estudos. A composição da floresta, biomassa e o histórico de exploração da área demonstraram que a fisionomia estudada apresenta-se em estádio secundário médio de regeneração avançando para uma condição mais tardia.

\footnotetext{
' Public University of Campinas, Campinas, São Paulo, Brazil

2 University of Sao Paulo, São Paulo, Brazil

${ }^{3}$ Botanical Institute - São Paulo, São Paulo, Brazil
} 


\section{INTRODUCTION}

The Brazilian Atlantic Forest (AF) is the second largest tropical moist forest of South America, covering, initially, ca. 1.450 million $\mathrm{km} 2$ (I7\%) of the country (JOLY et al., 2014) and approximately $80 \%$ of the São Paulo State (JOLY et al., 1999). The Atlantic Forest is considered the oldest Brazilian forest (RIZZINI, 1997), characterized by its high biodiversity and endemism and a hotspot for biodiversity conservation (MYERS et al., 2000), due land use change dating back to European's settlement (RIBEIRO et al., 2009). According to Malhi et al. (20/4), many human-modified tropical forest landscapes are complex and highly heterogeneous and must be better understood in order to develop efficient strategies for conservation.

In São Paulo State, only $15.3 \%$ of original forest cover remains (SOSMA/INPE, 20I3). The majority of these remnants are found in the mountainous region of southeast near the shore, where is also located the largest Conservation Unit of the Atlantic Forest, the Serra do Mar State Park (GALINDO-LEAL; CÂMARA, 2005). Nowadays, Atlantic Forest is mainly composed of isolated patches of small forests fragments, surrounded by pastures, agricultural fields and urbanization, and only few larger fragments remains (RIBEIRO et al., 2009). Hobbs et al. (2009) highlighted that the effect of human activities in the forest and the surrounds could promote the development of new ecosystems which differ in composition and/or function from present and past systems, especially as a consequence of changing species distributions and environmental alteration. In the last three decades, many studies have been devoted to Atlantic forest, firstly focused in fauna and flora's diversity and, more recently, focusing on the functionality of the forest and carbon stock (VIEIRA et al., 2008, JOLY et al., 20I4).

The Brazilian Atlantic Forest comprises five main forest physiognomies - Dense Ombrophilous, Open Ombrophilous, Mixed Ombrophilous, Seasonal Semideciduous and Seasonal Deciduous Forests (JOLY et al., 20I4). According to IBGE (20I2), the dominant vegetation in the state of São Paulo is classified as Ombrophilous Dense Forest and its subdivisions based on altimetry. The one which covers the Serra do Mar and the Atlantic Plateau, with altitudes between 500 and $1,500 \mathrm{~m}$, is classified as Montane Ombrophilous Dense Forest $(\mathrm{Dm})$ and is partially protected by the Serra do Mar State Park (SMSP). This forest formation, composition, and functionality is strongly influenced by environmental conditions, especially rainfall, temperature and the frequency of fog (ALVES et al., 20I0; VIEIRA et al., 20I I). The hillsides of Serra do Mar act as a moisture barrier against the sea breeze, which combined with the cold fronts and the South Atlantic Convergence Zone, resulting in an increase in precipitation rates, known as orographic rain (OLIVEIRA-FILHO; FONTES, 2000).

High altitudes environments, with colder and moister climates, tend to have lower temperatures and formation of mists and fog (BRUIJNZEEL; VENEKLAAS, 1998). Furthermore, these environmental conditions lead to the accumulation of large quantities of organic matter (SOETHE et al., 2008). In general, montane forests have higher density of individuals, lower diversity of species and families and, vascular and avascular epiphytic, compared with other tropical forests at lower altitudes (HAMILTON et al., 1995; LIEBERMAN et al., 1996). Alves et al. (2010) found an increase of aboveground biomass along of altitudinal gradient in the Ombrophilous Dense Atlantic Forest, as well as litter accumulation and carbon and nitrogen stocks, both above and below ground (VIEIRA et al., 20I I). In the montane forests, the environmental conditions are imposed by higher altitude belts and theirs species could be more sensitive to current changes in the global climate, suggesting an evidence of biodiversity loss a near future (SILVA; TABARELLI, 2000).

Phytosociological analysis and estimation of biomass and carbon stock of a plant community are essential for ecological study, evaluation of successional status and useful for carbon flow measurements between the forest and the atmosphere, resulting from the balance between gain through photosynthesis and loss through respiration and mortality (KEELING; PHILLIPS, 2007). Related to aboveground biomass, Melillo et al. (1993) suggested that the tropical forest has an important function on carbon exchange between atmosphere and terrestrial vegetation (36\%), but there are still scarce data about the stock of carbon. Biomass and carbon stock can be calculated in a non-destructive manner by using allometric equations (CHAVE et al., 2005), which vary according to plant physiognomy. The total aboveground biomass is strongly influenced by the canopy height variation, wood density, and community plant composition, as well as successional stage of the forest, ecological interactions, climate and soil conditions (CHAVE et al., 2005; VIEIRA et al., 2008; ALVES et al., 20I0).

The main aim of this study was to characterize the tree species composition and aboveground biomass of a community located in the footprint area of an Eddy covariance flux tower (KLJUN et al., 2002) in the Montane 
Ombrophilous Dense Forest, in the Santa Virginia Nucleus of Serra do Mar State Park. The plot studied had suffered clearcut for charcoal production more than 40 years ago, and since then, there is no more reported disturbance. This past disturbance influenced the observed ecological and biodiversity parameters. The long term carbon and water monitoring is fundamental for a better understanding of forest functionality, especially in Tropical Forests and climate change scenarios. In this aspect, the vegetation inventory is essential to characterize the plot status of succession and carbon flux, as plants are the largest biomass component in the ecosystem (FERSTER et al., 2015).

\section{MATERIAL AND METHODS}

\section{Study area}

This study was carried out in Ribeirão da Casa de Pedra watershed -Santa Virgínia Nucleus, Serra do Mar State Park - Ubatuba, SP, more specifically in the footprint area of the flux tower installed in 2007 (23. $17^{\prime}$ $23^{\circ} 24^{\prime} \mathrm{S}$ and $45^{\circ} 03^{\prime}-45^{\circ} \mathrm{II}$ 'W; I,020 m of altitude; http:// www.fluxdata.org:8080/SitePages/sitelnfo.aspx?BRAfs - Figure I). Santa Virgínia Nucleus at Serra do Mar State Park extending over an area of 17,000 hectares and altitude range from 740 to $1,600 \mathrm{~m}$ (INSTITUTO FLORESTAL, 2010) is considered an important area in biological and cultural aspects. Soils in the Serra do Mar State Park are classified as Inceptisol (USDA, 1999) with approximately $60 \%$ of sand, $20 \%$ of silt and $20 \%$ of clay (Alves et al., 2010). Moreover, they are characterized by the presence of shallow soils, geologically ancient, welldrained, low $\mathrm{pH}$, high phosphorus concentration and aluminum saturation compared to lower altimetric sites (SCARANELLO et al., 20I I; MARTINS, 20I0).

The forest area is characterized by average litter accumulation of $7.2 \mathrm{Mg} \mathrm{ha-1} \cdot \mathrm{yr}^{-1}$, relatively well distributed along the year (AIDAR et al., unpublished data) in the surface layers of the soil, which also contains the largest nutritional reserves (VIEIRA et al., 20I I). According to Köppen (1948), the regional climate can be classified as Cwa - temperate and tropical climate, with hot and wet summers, and slightly dry winters. The average minimum temperature is $10.6{ }^{\circ} \mathrm{C}$, the maximum of $26.1^{\circ} \mathrm{C}$ (MARTINS, 20I0) and the average annual rainfall of $2,200 \mathrm{~mm}$ with the wettest months being December, January and February (SIEGLOCH; FROEHLICH; SPIES, 20I2).

Veloso et al. (1991) classified the vegetation in the plot area as Montane Ombrophilous Dense Forest.
Tabarelli and Mantovani (1999) reported that as a result of land occupation and logging activities in the 1960's, the current forest vegetation is a mosaic with different successional stages (primary and secondary), pastures and Eucalyptus spp. plantations. Medeiros and Aidar (20II) found a physiognomy composed by large trees and uniform canopy covering $80 \%$ of the Ribeirão da Casa de Pedra watershed in Santa Virginia Nucleus.

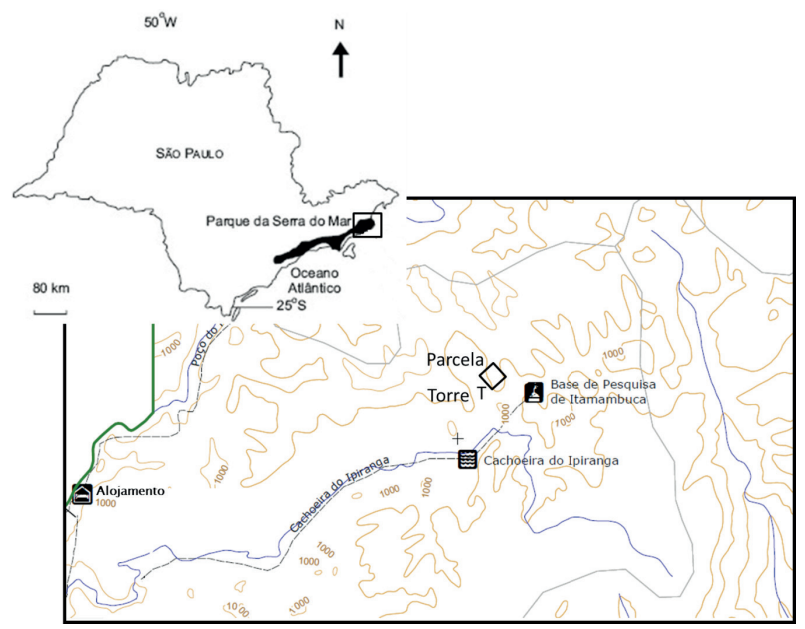

FIGURE I Maps showing the location of the region (Ubatuba, SP) and study area in Santa Virgínia Nucleus - Serra do Mar State Park. The symbol $(\diamond)$ indicate the sampled plot - Plot Torre (T).

\section{Vegetation Sampling}

The phytosociological survey was performed using the plot method (MUELLER-DOMBOIS; ELLENBERG, 1974). The sampling site is located in the flux tower footprint at I,020 m of altitude. The $100 \times 100 \mathrm{~m}$ (I ha) plot was divided into 25 blocks of $20 \times 20 \mathrm{~m}$. All living trees with stem diameter (one or multiple) at $1.30 \mathrm{~m}$ above the ground $(\mathrm{DBH}) \geq 4.8 \mathrm{~cm}$ were included in the sample. The approximate height (from the base to the highest branch) was acquired either with a clinometer or by comparison with known height, to define the average height. The plant material was collected in the reproductive or vegetative stage (FIDALGO; BONONI, 1984) and identified by using a specialized bibliography and comparisons with specimens deposited in herbarium collections from São Paulo ("SPSF - Instituto Florestal") and Campinas ("UEC - Universidade Estadual de Campinas"). The floristic list was prepared according to the classification system proposed in APG III (2009), and the confirmation and updating of species names and authors were accomplished by consulting the REFLORA - Flora do Brasil 2020 - LEFB database (FLORA DO BRASIL 2020, 2016). Materials with flowers, fruit, or 
vegetative were incorporated into the UEC Herbarium of the University of Campinas and the codes of the incorporated exsiccates can be found on the Herbarium of the State University of Campinas (UEC) available in the speciesLink Project (CRIA, 2016).

Estimates of the vegetation aboveground biomass (AGB) were carried out to complement the characterization of the study area and it is an useful knowledge to evaluate the carbon fluxes between aboveground forest ecosystems and the atmosphere (CHAVE et al., 2005). The model chosen for moist forest stands was based on Chave et al. (2005), the same employed by Medeiros and Aidar (20II) in other plot at Serra do Mar State Park (Equation I). The wood density values were extracted from Chave et al. (2006), when it was not available for one specific species, it was used the average of the genus or family. The allometric equations for palm trees (Equation 2) and ferns (Equation 3) were based on other authors (NASCIMENTO; LAURANCE, 2002; TIEPOLO et al., 2002, respectively). Lianas, bamboos, and epiphytes were not included. Where, $\rho=$ wood specific density $\left(\mathrm{g} \cdot \mathrm{cm}^{-3}\right)$ (CHAVE et al. 2006, ALVES et al. 20l0), $\mathrm{DBH}=$ diameter at $1.30 \mathrm{~m}$ height from the ground $(\mathrm{cm})$, $\mathrm{H}=$ height $(\mathrm{m})$, exp = exponent applied to the base, $\mathrm{ln}=$ natural logarithm.

$$
\begin{aligned}
& A G B=\exp \left[-2.977+\ln \left(\rho \cdot \mathrm{DBH}^{2} \cdot \mathrm{H}\right)\right] \\
& \mathrm{AGB}=\left\{\exp \left[0.9285 \cdot \ln \left(\mathrm{DBH}^{2}\right)+5.7236\right] \cdot 1.05001\right\} \cdot 10^{-3} \\
& \mathrm{AGB}=-4266348 \cdot\{1-[2792284 \cdot \exp (-0.313677 \cdot H)]\}^{-1}
\end{aligned}
$$

List of 20 most important species, families and phytosociol

\begin{tabular}{|c|c|c|c|c|c|c|c|}
\hline Species & Family & NInd & RelDe & RelFr & IV & CV & SG \\
\hline Alchornea triplinervia (Spreng.) Müll. Arg. & Euphorbiaceae & 245 & 15.35 & 3.29 & 45.55 & 42.21 & $\mathrm{ES}$ \\
\hline Euterpe edulis Mart. & Arecaceae & 267 & 16.73 & 3.59 & 32.22 & 28.60 & $\mathrm{ES}$ \\
\hline Cyatheaceae n.i & Cyatheaceae & 117 & 7.33 & 2.84 & $|4.6|$ & 11.70 & $\mathrm{ES}$ \\
\hline Miconia cabucu Hoehne & Melastomataceae & 44 & 2.76 & 2.69 & 7.79 & 5.11 & ES \\
\hline Tibouchina pulchra Cogn. & Melastomataceae & 23 & 1.44 & 1.79 & 6.91 & 5.12 & $\mathrm{ES}$ \\
\hline Myrcia spectabilis DC. & Myrtaceae & 47 & 2.94 & 2.69 & 6.90 & 4.21 & LS \\
\hline Licania hoehnei Pilg. & Chrysobalanaceae & 24 & 1.50 & 1.35 & 6.32 & 4.97 & LS \\
\hline Guapira opposita (Vell.) Reitz & Nyctaginaceae & 35 & 2.19 & 2.39 & 6.20 & 3.81 & $\mathrm{ES}$ \\
\hline Piptocarpha macropoda (DC.) Baker & Asteraceae & 17 & 1.07 & 1.64 & 5.87 & 4.22 & $\mathrm{Pl}$ \\
\hline Solanum cinnamomeum Sendtn. & Solanaceae & 28 & 1.75 & 1.49 & 5.65 & 4.15 & $\mathrm{PI}$ \\
\hline Myrcia splendens (Sw.) DC. & Myrtaceae & 28 & 1.75 & 2.24 & 5.03 & 2.78 & LS \\
\hline Schefflera angustissima (Marchal) Frodin & Araliaceae & 20 & 1.25 & 1.79 & 5.02 & 3.22 & ES \\
\hline Bathysa australis (A.St.-Hil.) K.Schum. & Rubiaceae & 28 & 1.75 & 1.94 & 4.75 & 2.81 & LS \\
\hline Guatteria australis A. St.-Hil. & Annonaceae & 27 & 1.69 & 2.24 & 4.45 & 2.21 & LS \\
\hline Ocotea dispersa (Nees and Mart.) Mez & Lauraceae & 25 & 1.57 & 2.09 & 4.33 & 2.24 & LS \\
\hline Mollinedia argyrogyna Perkins & Monimiaceae & 18 & 1.13 & 1.49 & 4.19 & 2.71 & ES \\
\hline Sloanea hirsuta (Schott) Planch. ex Benth. & Elaeocarpaceae & 12 & 0.75 & 1.21 & 3.8 & 2.60 & LS \\
\hline Cryptocarya mandioccana Meisn. & Lauraceae & 20 & 1.25 & 1.94 & 3.64 & 1.71 & LS \\
\hline Chrysophyllum viride Mart. and Eichler & Sapotaceae & 5 & 0.31 & 0.75 & 3.49 & 2.74 & LS \\
\hline Inga lanceifolia Benth. & Fabaceae & 20 & 1.25 & 1.79 & 3.43 & 1.64 & LS \\
\hline
\end{tabular}
Mar State Park. NInd - Number of individuals, RelDe - Relative density, RelFr - Relative frequency, IV - Importance value, CV Coverage value, SG. - Successional group (PI - Pioneer, ES - Early secondary, LS - Late secondary and NC - Not characterized).

\section{Data Analysis}

Stem density, absolute and relative dominance (Basal area), absolute and relative frequency, importance, coverage and diversity (Shannon's diversity, H'; Pielou's evenness, J) indexes were calculated using FITOPAC 2.I software (SHEPHERD, 20I0; PIELOU, 1975). Individuals were grouped by diameter and height classes to evaluate the forest structure. In addition, the species were classified into the successional groups proposed by Gandolfi et al. (1995), especially considering the survival and germination in the light, as pioneer, early secondary and late secondary. Comparisons with the literature (AIDAR et al., 2003; GOMES et al., 20II; MEDEIROS AND AIDAR, 20II; PADGURSCHI et al., 20I I) were used for the species definition in each of the ecological groups.

\section{RESULTS AND DISCUSSION}

Of a total of 1,704 trees found, I,596 (93.7 $\%)$ were identified, 75 (4.4\%) were unidentified and 33 (1.9\%) correspond to standing dead individuals. The identified individual trees were distributed in 143 species belonging to 38 botanical families (Table I and 2), including 258 palms (Euterpe edulis - Arecaceae) and II 7 ferns represented by Cyatheaceae family.

The average and maximum values of diameter were $12.8 \mathrm{~cm}$ and $108.2 \mathrm{~cm}$, respectively. The maximum height were $35.5 \mathrm{~m}$ and average of $10.3 \mathrm{~m}$. Stem density values $\left(\mathrm{I}, 704\right.$ ind $\left.\cdot \mathrm{ha}^{-1}\right)$ and basal area $\left(3 \mathrm{I} .9 \mathrm{~m}^{2} \cdot \mathrm{ha}^{-1}\right)$ were values $\left(1,704\right.$ ind $\left.\cdot \mathrm{ha}^{-1}\right)$ and basal area $\left(31.9 \mathrm{~m}^{2} \cdot \mathrm{ha}^{-}\right)$were Species Family NInd RelDe RelFr IV CV $\quad$ SG linervia (Spreng.) Müll. Arg. Euphorbiaceae Cyatheacea Melastomatacea 
TABLE 2 List of species, families and phytosociological parameters in the Montane Ombrophilous Dense Forest, Serra do Mar State Park. NInd - Number of individuals, RelDe - Relative density, RelFr - Relative frequency, IV - Importance value, CV - Coverage value, SG. - Successional group (PI - Pioneer, ES - Early secondary, LS - Late secondary and NC - Not characterized).

\begin{tabular}{|c|c|c|c|c|c|c|c|c|}
\hline Species & Family & NInd & RelDe & RelFr & IV & $\mathrm{CV}$ & SG & Voucher \\
\hline Alchornea triplinervia (Spreng.) Müll. Arg. & Euphorbiaceae & 245 & 15.35 & 3.29 & 45.55 & 42.21 & ES & UEC 160360 \\
\hline Euterpe edulis Mart. & Arecaceae & 267 & 16.73 & 3.59 & 32.22 & 28.60 & ES & - \\
\hline Cyatheaceae n.i & Cyatheaceae & 117 & 7.33 & 2.84 & 14.61 & 11.70 & ES & - \\
\hline Miconia cabucu Hoehne & Melastomataceae & 44 & 2.76 & 2.69 & 7.79 & 5.11 & ES & UEC 160402 \\
\hline Tibouchina pulchra Cogn. & Melastomataceae & 23 & 1.44 & 1.79 & 6.91 & 5.12 & ES & - \\
\hline Myrcia spectabilis DC. & Myrtaceae & 47 & 2.94 & 2.69 & 6.90 & 4.21 & LS & UEC 160385 \\
\hline Licania hoehnei Pilg. & Chrysobalanaceae & 24 & 1.50 & 1.35 & 6.32 & 4.97 & LS & UEC 160356 \\
\hline Guapira opposita (Vell.) Reitz & Nyctaginaceae & 35 & 2.19 & 2.39 & 6.20 & 3.81 & ES & UEC 160414 \\
\hline Piptocarpha macropoda (DC.) Baker & Asteraceae & 17 & 1.07 & 1.64 & 5.87 & 4.22 & $\mathrm{PI}$ & UEC 160347 \\
\hline Solanum cinnamomeum Sendtn. & Solanaceae & 28 & 1.75 & 1.49 & 5.65 & 4.15 & $\mathrm{PI}$ & UEC I60448 \\
\hline Myrcia splendens (Sw.) DC. & Myrtaceae & 28 & 1.75 & 2.24 & 5.03 & 2.78 & LS & UEC 160383 \\
\hline Schefflera angustissima (Marchal) Frodin & Araliaceae & 20 & 1.25 & 1.79 & 5.02 & 3.22 & ES & UEC 160337 \\
\hline Bathysa australis (A.St.-Hil.) K.Schum. & Rubiaceae & 28 & 1.75 & 1.94 & 4.75 & 2.81 & LS & UEC 160429 \\
\hline Guatteria australis A. St.-Hil. & Annonaceae & 27 & 1.69 & 2.24 & 4.45 & 2.21 & LS & UEC I6034 I \\
\hline Ocotea dispersa (Nees and Mart.) Mez & Lauraceae & 25 & 1.57 & 2.09 & 4.33 & 2.24 & LS & UEC 160327 \\
\hline Mollinedia argyrogyna Perkins & Monimiaceae & 18 & 1.13 & 1.49 & 4.19 & 2.71 & ES & UEC 160408 \\
\hline Sloanea hirsuta (Schott) Planch. ex Benth. & Elaeocarpaceae & 12 & 0.75 & 1.21 & 3.8 & 2.60 & LS & UEC 160358 \\
\hline Cryptocarya mandioccana Meisn. & Lauraceae & 20 & 1.25 & 1.94 & 3.64 & 1.71 & LS & UEC 160333 \\
\hline Chrysophyllum viride Mart. and Eichler & Sapotaceae & 5 & 0.31 & 0.75 & 3.49 & 2.74 & LS & UEC 160443 \\
\hline Inga lanceifolia Benth. & Fabaceae & 20 & 1.25 & 1.79 & 3.43 & 1.64 & LS & UEC 160317 \\
\hline Inga marginata Willd. & Fabaceae & 8 & 0.5 & 0.6 & 3.38 & 2.79 & ES & UEC 160320 \\
\hline Calyptranthes strigipes O. Berg & Myrtaceae & 12 & 0.75 & 1.05 & 3.38 & 2.34 & LS & UEC 160364 \\
\hline Hyeronima alchorneoides Allemão & Phyllanthaceae & 12 & 0.75 & 1.35 & 3.32 & 1.98 & ES & UEC I6042 I \\
\hline Mollinedia schottiana (Spreng.) Perkins & Monimiaceae & 17 & 1.07 & 1.64 & 3.27 & 1.62 & LS & UEC 160407 \\
\hline Pouteria caimito (Ruiz and Pav.) Radlk. & Sapotaceae & 10 & 0.63 & 1.21 & 3.19 & 1.99 & LS & UEC 160442 \\
\hline Cupania vernalis Cambess. & Sapindaceae & 14 & 0.88 & 1.64 & 3.1 & 1.45 & ES & UEC 160438 \\
\hline Calyptranthes lucida Mart. ex DC. & Myrtaceae & 16 & 1,00 & 1.05 & 3.03 & 1.99 & LS & UEC 160367 \\
\hline Cryptocarya saligna Mez & Lauraceae & 12 & 0.75 & 1.64 & 2.88 & 1.03 & LS & UEC 160332 \\
\hline Ocotea catharinensis Mez & Lauraceae & 14 & 0.88 & 1.2 & 2.87 & 1.67 & LS & UEC 160325 \\
\hline Matayba guianensis Aubl. & Sapindaceae & 19 & 1.19 & 1.2 & 2.77 & 1.57 & LS & UEC 160440 \\
\hline Eugenia prasina O. Berg & Myrtaceae & 14 & 0.88 & 0.9 & 2.68 & 1.79 & LS & UEC 160370 \\
\hline Psychotria vellosiana Benth. & Rubiaceae & 17 & 1.07 & 1.2 & 2.58 & 1.39 & $\mathrm{NC}$ & UEC I6043 I \\
\hline Inga sessilis (Vell.) Mart. & Fabaceae & 9 & 0.56 & 0.75 & 2.36 & 1.62 & ES & UEC 160319 \\
\hline Cinnamomum sp.I Schaeff. & Lauraceae & 13 & 0.81 & 1.2 & 2.35 & 1.16 & LS & UEC 160335 \\
\hline Myrsine gardneriana A. DC. & Primulaceae & 9 & 0.56 & 1.35 & 2.28 & 0.93 & ES & UEC 160423 \\
\hline Hirtella hebeclada Moric. ex DC. & Chrysobalanaceae & 9 & 0.56 & 1.2 & 2.16 & 0.97 & LS & UEC 160357 \\
\hline Cordia sellowiana Cham. & Boraginaceae & 11 & 0.69 & 1.2 & 2.13 & 0.93 & ES & UEC 160344 \\
\hline Guapira nitida (Mart. ex J.A.) Lundell & Nyctaginaceae & 8 & 0.5 & 0.24 & 2.12 & 1.08 & ES & UEC 160415 \\
\hline Psychotria sp.I L. & Rubiaceae & 13 & 0.81 & 1.05 & 2.01 & 0.96 & $\mathrm{NC}$ & UEC 160434 \\
\hline Guarea macrophylla Vahl & Meliaceae & 8 & 0.5 & 1.05 & 1.92 & 0.87 & LS & UEC 160397 \\
\hline Allophylus petiolulatus Radlk. & Sapindaceae & 10 & 0.63 & 0.75 & 1.9 & 1.16 & ES & UEC 160439 \\
\hline Miconia aff. latecrenata Triana & Melastomataceae & 8 & 0.50 & 0.90 & 1.80 & 0.90 & ES & UEC 160403 \\
\hline Eugenia oblongata O. Berg & Myrtaceae & 7 & 0.44 & 0.90 & 1.75 & 0.85 & LS & UEC 160375 \\
\hline Myrcia guianensis (Aubl.) DC. & Myrtaceae & 9 & 0.56 & 0.90 & 1.72 & 0.82 & LS & - \\
\hline Nectandra oppositifolia Nees & Lauraceae & 8 & 0.5 & 0.90 & 1.71 & 0.81 & LS & UEC 160330 \\
\hline Vernonanthura puberula (Less.) H.Rob. & Asteraceae & 4 & 0.25 & 0.45 & 1.59 & 1.14 & $\mathrm{PI}$ & UEC 160346 \\
\hline Myrsine leuconeura Mart. & Primulaceae & 8 & 0.5 & 0.75 & 1.58 & 0.83 & ES & UEC 160422 \\
\hline Casearia obliqua Spreng. & Salicaceae & 9 & 0.56 & 0.6 & 1.54 & 0.94 & LS & UEC 160437 \\
\hline Cedrela fissilis Vell. & Meliaceae & 7 & 0.44 & 0.75 & 1.52 & 0.77 & ES & UEC 160396 \\
\hline Miconia atlantica Caddah \& R.Goldenb. & Melastomataceae & 6 & 0.38 & 0.9 & 1.49 & 0.60 & ES & UEC 160404 \\
\hline Amaioua intermedia Mart. ex Schult. and Schult.f. & Rubiaceae & 6 & 0.38 & 0.9 & 1.42 & 0.53 & ES & UEC 160428 \\
\hline Annona sylvatica A. St.-Hil. & Annonaceae & 7 & 0.44 & 0.75 & 1.32 & 0.57 & ES & UEC 160340 \\
\hline Dendropanax monogynus (Vell.) Seem. & Araliaceae & 2 & 0.13 & 0.30 & 1.23 & 0.93 & $\mathrm{NC}$ & UEC 160336 \\
\hline Cabralea canjerana (Vell.) Mart. & Meliaceae & 5 & 0.31 & 0.75 & 1.18 & 0.43 & LS & UEC 160393 \\
\hline Heisteria silvianii Schwacke & Olacaceae & 5 & 0.31 & 0.6 & 1.11 & 0.51 & LS & UEC 160416 \\
\hline Psychotria suterella Müll. Arg. & Rubiaceae & 7 & 0.44 & 0.6 & 1.11 & 0.51 & $\mathrm{NC}$ & UEC 160433 \\
\hline
\end{tabular}




\begin{tabular}{|c|c|c|c|c|c|c|c|c|}
\hline Species & Family & NInd & RelDe & RelFr & IV & $\mathrm{CV}$ & SG & Voucher \\
\hline Ocotea aciphylla (Nees and Mart.) Mez & Lauraceae & 4 & 0.25 & 0.6 & 1.08 & 0.49 & LS & UEC 160326 \\
\hline Vernonia sp.I Schreb. & Asteraceae & 4 & 0.25 & 0.45 & 1.07 & 0.62 & $\mathrm{PI}$ & - \\
\hline Sloanea guianensis (Aubl.) Benth. & Elaeocarpaceae & 4 & 0.25 & 0.6 & 1.02 & 0.43 & LS & UEC 160359 \\
\hline Trichilia pallida Sw. & Meliaceae & 7 & 0.44 & 0.45 & 1.02 & 0.58 & LS & UEC 160395 \\
\hline Leandra mosenii Cogn. & Melastomataceae & 4 & 0.25 & 0.6 & 0.98 & 0.38 & ES & - \\
\hline Myrceugenia myrcioides (Cambess.) O. Berg & Myrtaceae & 6 & 0.38 & 0.45 & 0.98 & 0.53 & LS & UEC 160378 \\
\hline Mollinedia oligantha Perkins & Monimiaceae & 3 & 0.19 & 0.45 & 0.95 & 0.50 & $\mathrm{NC}$ & UEC 160406 \\
\hline Piper cernuum Vell. & Piperaceae & 4 & 0.25 & 0.61 & 0.93 & 0.33 & ES & UEC 160419 \\
\hline Cordia trichoclada DC. & Boraginaceae & 4 & 0.25 & 0.61 & 0.93 & 0.33 & ES & UEC 160343 \\
\hline Annona dolabripetala Raddi & Annonaceae & 3 & 0.19 & 0.31 & 0.83 & 0.53 & ES & UEC 160339 \\
\hline Marcgravia polyantha Delpino & Marcgraviaceae & 5 & 0.31 & 0.31 & 0.81 & 0.51 & $\mathrm{NC}$ & UEC 160391 \\
\hline Marlierea silvatica (O.Berg) Kiaersk. & Myrtaceae & 3 & 0.19 & 0.45 & 0.79 & 0.34 & LS & UEC 160376 \\
\hline Aniba firmula (Nees and Mart.) Mez & Lauraceae & 4 & 0.25 & 0.45 & 0.78 & 0.33 & LS & UEC 160324 \\
\hline Daphnopsis fasciculata (Meisn.) Nevling & Thymelaeaceae & 4 & 0.25 & 0.45 & 0.78 & 0.33 & LS & - \\
\hline Citronella paniculata (Mart.) R.A. Howard & Cardiopteridaceae & 4 & 0.25 & 0.45 & 0.76 & 0.31 & LS & UEC 160351 \\
\hline Aureliana fasciculata (Vell.) Sendtn. & Solanaceae & 6 & 0.38 & 0.30 & 0.76 & 0.46 & ES & UEC 160449 \\
\hline Rudgea vellerea Müll. Arg. & Rubiaceae & 4 & 0.25 & 0.45 & 0.74 & 0.29 & LS & UEC 160435 \\
\hline Maytenus sp.I & Celastraceae & 3 & 0.19 & 0.45 & 0.73 & 0.28 & LS & UEC 160353 \\
\hline Casearia paranaensis Sleumer & Salicaceae & 3 & 0.19 & 0.45 & 0.73 & 0.28 & LS & UEC 160436 \\
\hline Mollinedia glabra (Spreng.) Perkins & Monimiaceae & 5 & 0.31 & 0.31 & 0.72 & 0.42 & NC & UEC I604II \\
\hline Myrcia sp.I & Myrtaceae & 3 & 0.19 & 0.45 & 0.7 & 0.26 & LS & UEC 160390 \\
\hline Myrceugenia miersiana (Gardner) D. Legrand and Kausel & Myrtaceae & 3 & 0.19 & 0.45 & 0.68 & 0.23 & LS & - \\
\hline Piptocarpha sellowii (Sch. Bip.) Baker & Asteraceae & 2 & 0.13 & 0.31 & 0.67 & 0.38 & $\mathrm{PI}$ & UEC 160348 \\
\hline Eugenia verticillata (Vell.) Angely & Myrtaceae & 3 & 0.19 & 0.45 & 0.67 & 0.22 & LS & UEC 160374 \\
\hline Campomanesia guaviroba (DC.) Kiaersk. & Myrtaceae & 3 & 0.19 & 0.31 & 0.55 & 0.25 & ES & UEC 160369 \\
\hline Ocotea glaziovii Mez & Lauraceae & 3 & 0.19 & 0.31 & 0.54 & 0.25 & LS & UEC 160329 \\
\hline Simira viridiflora (Allemão and Saldanha) Steyerm. & Rubiaceae & 3 & 0.19 & 0.31 & 0.54 & 0.24 & NC & UEC 160432 \\
\hline Mollinedia ovata Ruiz and Pav. & Monimiaceae & 2 & 0.13 & 0.31 & 0.53 & 0.24 & $\mathrm{NC}$ & UEC 160412 \\
\hline Myrcia freyreissiana (O. Berg) Kiaersk. & Myrtaceae & 3 & 0.19 & 0.31 & 0.52 & 0.22 & LS & UEC 160388 \\
\hline Chrysophyllum inornatum Mart. & Sapotaceae & 3 & 0.19 & 0.31 & 0.52 & 0.22 & LS & UEC 160444 \\
\hline Symplocos estrellensis Casar. & Symplocaceae & 3 & 0.19 & 0.31 & 0.52 & 0.23 & ES & UEC 16045I \\
\hline Phytolacca dioica L. & Phytolaccaceae & 2 & 0.13 & 0.31 & 0.51 & 0.21 & $\mathrm{NC}$ & UEC 160426 \\
\hline Ilex microdonta Reissek & Aquifoliaceae & 2 & 0.13 & 0.31 & 0.5 & 0.2 & LS & UEC 160338 \\
\hline Ocotea daphnifolia (Meisn.) Mez & Lauraceae & 2 & 0.13 & 0.31 & 0.49 & 0.19 & LS & UEC 161508 \\
\hline Tachigali sp.I Aubl. & Fabaceae & 2 & 0.13 & 0.31 & 0.48 & 0.18 & $\mathrm{NC}$ & UEC 160323 \\
\hline Cryptocarya aschersoniana Mez & Lauraceae & 2 & 0.13 & 0.15 & 0.48 & 0.33 & LS & UEC 160334 \\
\hline Myrciaria floribunda (H.West ex Willd.) O.Berg & Myrtaceae & 1 & 0.06 & 0.15 & 0.48 & 0.33 & LS & UEC 16038I \\
\hline Matayba sp. I Aubl. & Sapindaceae & 2 & 0.13 & 0.31 & 0.48 & 0.18 & LS & UEC 161512 \\
\hline Myrcia tijucensis Kiaersk. & Myrtaceae & 2 & 0.13 & 0.31 & 0.47 & 0.17 & LS & UEC 160389 \\
\hline Pouteria gardneri (Mart. and Miq.) Baehni & Sapotaceae & 2 & 0.13 & 0.31 & 0.47 & 0.17 & LS & UEC 160445 \\
\hline Piptocarpha axillaris (Less.) Baker & Asteraceae & 1 & 0.06 & 0.15 & 0.46 & 0.31 & $\mathrm{PI}$ & UEC 160350 \\
\hline Mollinedia engleriana Perkins & Monimiaceae & 2 & 0.13 & 0.31 & 0.46 & 0.16 & LS & UEC 160410 \\
\hline Mollinedia acutissima Perkins & Monimiaceae & 2 & 0.13 & 0.31 & 0.46 & 0.16 & $\mathrm{NC}$ & UEC 160413 \\
\hline Symplocos sp. I Jacq. & Symplocaceae & 2 & 0.13 & 0.31 & 0.46 & 0.16 & ES & UEC 160452 \\
\hline Trichilia pallens C. DC. & Meliaceae & 2 & 0.13 & 0.31 & 0.46 & 0.16 & LS & UEC 161510 \\
\hline Trichilia silvatica C. DC & Meliaceae & 2 & 0.13 & 0.31 & 0.45 & 0.15 & LS & - \\
\hline Maytenus sp. 2 Molina & Celastraceae & 3 & 0.19 & 0.15 & 0.45 & 0.31 & LS & UEC 160354 \\
\hline Calyptranthes sp. I Sw. & Myrtaceae & 2 & 0.13 & 0.31 & 0.45 & 0.15 & LS & UEC 160366 \\
\hline Aniba viridis Mez & Lauraceae & 2 & 0.13 & 0.31 & 0.45 & 0.15 & LS & - \\
\hline Mollinedia blumenaviana Perkins & Monimiaceae & 2 & 0.13 & 0.31 & 0.44 & 0.14 & ES & UEC 160409 \\
\hline Stylogyne Ihotzkyana (A.DC.) Mez & Primulaceae & 2 & 0.13 & 0.31 & 0.44 & 0.14 & $\mathrm{NC}$ & UEC 160425 \\
\hline Cecropia glaziovii Snethl. & Urticaceae & $\mathrm{I}$ & 0.06 & 0.15 & 0.42 & 0.27 & $\mathrm{PI}$ & - \\
\hline Sessea sp.I Ruiz and Pav. & Solanaceae & 1 & 0.06 & 0.15 & 0.42 & 0.27 & ES & UEC 160447 \\
\hline Myrtaceae sp.I Juss. & Myrtaceae & $\mathrm{I}$ & 0.06 & 0.15 & 0.41 & 0.26 & LS & - \\
\hline Byrsonima ligustrifolia A. Juss. & Malpighiaceae & 3 & 0.19 & 0.15 & 0.39 & 0.24 & ES & UEC 160392 \\
\hline Tapiria guianensis Aubl. & Anacardiaceae & 1 & 0.06 & 0.15 & 0.37 & 0.22 & ES & - \\
\hline Ardisia guianensis (Aubl.) Mez & Primulaceae & 1 & 0.06 & 0.15 & 0.34 & 0.19 & ES & - \\
\hline Matayba juglandifolia (Cambess.) Radlk. & Sapindaceae & 2 & 0.13 & 0.15 & 0.32 & 0.17 & LS & - \\
\hline Vernonanthura petiolaris (DC.) H. Rob. & Asteraceae & 1 & 0.06 & 0.15 & 0.28 & 0.13 & $\mathrm{PI}$ & - \\
\hline
\end{tabular}




\begin{tabular}{|c|c|c|c|c|c|c|c|c|}
\hline Species & Family & NInd & RelDe & RelFr & IV & $\mathrm{CV}$ & SG & Voucher \\
\hline Symplocos laxiflora Benth. & Symplocaceae & $\mathrm{I}$ & 0.06 & 0.15 & 0.26 & 0.11 & ES & UEC 160450 \\
\hline Terminalia januariensis DC. & Combretaceae & I & 0.06 & 0.15 & 0.25 & 0.11 & $\mathrm{NC}$ & - \\
\hline Inga laurina (Sw.) Willd. & Fabaceae & $\mathrm{I}$ & 0.06 & 0.15 & 0.25 & 0.11 & $\mathrm{NC}$ & UEC 160318 \\
\hline Nectandra grandiflora Nees & Lauraceae & 1 & 0.06 & 0.15 & 0.25 & 0.11 & LS & UEC 160331 \\
\hline Rhodostemonodaphne macrocalyx (Meisn.) Rohwer ex Madriñán & Lauraceae & $\mathrm{I}$ & 0.06 & 0.15 & 0.24 & 0.09 & LS & UEC 160328 \\
\hline Eugenia brevistyla D.Legrand & Myrtaceae & $\mathrm{I}$ & 0.06 & 0.15 & 0.24 & 0.09 & LS & UEC 160373 \\
\hline Myrceugenia campestris (DC.) D. Legrand and Kausel & Myrtaceae & $\mathrm{I}$ & 0.06 & 0.15 & 0.24 & 0.09 & LS & UEC 160380 \\
\hline Sapium glandulosum (L.) Morong & Euphorbiaceae & 1 & 0.06 & 0.15 & 0.23 & 0.08 & ES & UEC 160362 \\
\hline Campomanesia neriiflora (O. Berg) Nied. & Myrtaceae & 1 & 0.06 & 0.15 & 0.23 & 0.08 & LS & UEC 160368 \\
\hline Marlierea tomentosa Cambess. & Myrtaceae & 1 & 0.06 & 0.15 & 0.23 & 0.08 & ES & UEC 160377 \\
\hline Guarea kunthiana A. Juss. & Meliaceae & 1 & 0.06 & 0.15 & 0.23 & 0.08 & LS & UEC 160398 \\
\hline Quiina glaziovii Engl. & Ochnaceae & $\mathrm{I}$ & 0.06 & 0.15 & 0.23 & 0.08 & LS & UEC 160418 \\
\hline Cupania oblongifolia Mart. & Sapindaceae & 1 & 0.06 & 0.15 & 0.23 & 0.08 & LS & UEC I6044 I \\
\hline Byrsonima sp.I Rich. ex Kunth & Malpighiaceae & 1 & 0.06 & 0.15 & 0.23 & 0.08 & ES & UEC 161509 \\
\hline Myrtaceae sp. 2 Juss. & Myrtaceae & 1 & 0.06 & 0.15 & 0.22 & 0.07 & LS & - \\
\hline Vantanea compacta (Schnizl.) Cuatrec. & Humiriaceae & 1 & 0.06 & 0.15 & 0.22 & 0.07 & LS & UEC 160315 \\
\hline Machaerium sp.I Pers. & Fabaceae & $\mathrm{I}$ & 0.06 & 0.15 & 0.22 & 0.07 & LS & UEC 16032I \\
\hline Pterocarpus rohrii Vahl & Fabaceae & $\mathrm{I}$ & 0.06 & 0.15 & 0.22 & 0.07 & LS & UEC 160322 \\
\hline Salacia elliptica (Mart. ex Schult.) G. Don & Celastraceae & 1 & 0.06 & 0.15 & 0.22 & 0.07 & ES & UEC 160355 \\
\hline Mabea piriri Aubl. & Euphorbiaceae & 1 & 0.06 & 0.15 & 0.22 & 0.07 & $\mathrm{NC}$ & UEC 16036I \\
\hline Eugenia cuprea (O.Berg) Nied. & Myrtaceae & 1 & 0.06 & 0.15 & 0.22 & 0.07 & LS & UEC 160372 \\
\hline Cedrela odorata L. & Meliaceae & 1 & 0.06 & 0.15 & 0.22 & 0.07 & ES & UEC 160394 \\
\hline Mollinedia oligotricha Perkins & Monimiaceae & 1 & 0.06 & 0.15 & 0.22 & 0.07 & NC & UEC 160405 \\
\hline Quiina magallano-gomesii Schwacke & Ochnaceae & $\mathrm{I}$ & 0.06 & 0.15 & 0.22 & 0.07 & LS & UEC 160417 \\
\hline Myrsine umbellata Mart. & Primulaceae & 1 & 0.06 & 0.15 & 0.22 & 0.08 & ES & UEC 160424 \\
\hline Posoqueria latifolia (Rudge) Roem. and Schult. & Rubiaceae & 1 & 0.06 & 0.15 & 0.22 & 0.07 & ES & UEC 160430 \\
\hline Nectandra puberula (Schott) Nees & Lauraceae & 1 & 0.06 & 0.15 & 0.22 & 0.07 & LS & UEC 161507 \\
\hline Eugenia excelsa O. Berg & Myrtaceae & $\mathrm{I}$ & 0.06 & 0.15 & 0.22 & 0.07 & LS & UEC 161511 \\
\hline
\end{tabular}

similar to those found in other studies also developed in the area (MEDEIROS; AIDAR, 20I I; ALVES et al., 20I0; PADGURSCHI et al., 20I I). Medeiros and Aidar (20I I), in a Montane Ombrophilous Dense Forest community of with more than 35 years of regeneration, found I,743.3 ind $\cdot \mathrm{ha}^{-1}$ and basal area of $28.5 \mathrm{~m}^{2} \cdot \mathrm{ha}^{-1}$, whereas Tabarelli and Mantovani (1999) in other patch of 40-year forest at $870 \mathrm{~m}$ to $\mathrm{I} .100 \mathrm{~m}$ obtained $2,735 \mathrm{ind} \cdot \mathrm{ha}^{-1}$ and 33.4 ind $\cdot \mathrm{ha}^{-1}$. Joly et al. (2012) compared all permanent plots of the Atlantic Rainforest along an altitudinal gradient in southeastern Brazil and obtained I,965 stems.ha ${ }^{-1}$ in a mature and preserved plot at Santa Virginia Nucleus, with higher percentage of palms compared to others phytophisiognomies. Lieberman et al. (1996) highlighted a positive relationship between tree density and altitude at Atlantic Forest and other tropical forests.

The Shannon diversity index $\mathrm{H}^{\prime}=3.7$ nats.ind. ${ }^{-1}$ and the evenness index $\mathrm{J}^{\prime}=0.7$, demonstrated that the community is diverse, but with uneven distribution of individuals by species, including species with a great number of individuals and species with only one representative. Other plots at Santa Virgínia Nucleus, located in different areas and with distinct historical use, showed similar indexes values as found in this study (both with $\mathrm{H}^{\prime}=3.6$ nats.ind.$^{-1}$ and $\mathrm{J}=0.7$ ) (PADGURSCHI et al., 20I I; MEDEIROS; AIDAR, 20II ). Padgurschi et al. (20I I) compared two plots with different history of human impact within the Serra do Mar State Park regarding to the floristic composition, forest structure and aboveground biomass, and they found lower biodiversity in the plot that suffered selective logging 40 years ago in comparison to the undisturbed site $\left(\mathrm{H}^{\prime}=3.72\right.$ nats.ind. ${ }^{-1}, \mathrm{~J}=0.7$ and $H^{\prime}=4,05$ nats.ind. $.^{-1}, J=0.8$, respectively; reduction in 40 tree species). Considering altitude, sites located in lower altitudes showed higher Shannon's diversity indexes (348 e $395 \mathrm{~m}$ altitude - H' = 4.5 nats.ind.$^{-1}$ - ROCHELLE et al., 20 I I) and confirmed the hypothesis that there is a trend to the reduction in diversity with the increased altitude, since the environmental conditions imposed by higher altitudes, such as temperature reduction, frequent fog's events and high rates of precipitation, could limit the occurrence of some species and reduce the diversity indexes (TABARELLI; MANTOVANI, 1999).

The families' dominance (basal area) in the study area, especially Arecaceae, Cyatheaceae, Fabaceae, Lauraceae, Myrtaceae and Rubiaceae were similar to the results obtained by Tabarelli and Mantovani (1999), Padgurschi et al. (20I I), Medeiros and Aidar (20I I) and 
Joly et al. (2012). Peixoto and Gentry (1990) pointed that Myrtaceae and Lauraceae families predominate in forest formations along the entire Atlantic coast, especially under the influence of fog or in the montane forests. The sum of these families resulted in approximately $54 \%$ of the individuals present in the sampling area and influenced the importance value and coverage value results. They are often found in Atlantic Forest, especially in montane formations, along with a low number of woody vines and a high abundance of epiphytes, ferns and bamboos (JOLY et al., 20l4; 20I2). The presence of the Monimiaceae family with 52 individuals and nine species, confirms the hypothesis of Peixoto (1987) and Padgurschi (2010), which observed the same species in a near plot at montane Forests in Serra do Mar State Park. In fact, there are many species similarities between this study and the Padgurschi et al. (201 I): Firstly, both had experienced a disruption for over 40 years ago and; secondly, both showed abundance of recognizable secondary species, like Inga spp., Miconia spp. and Alchornea triplinervia.

The Arecaceae family was represented only by Euterpe edulis, as also found by Padgurschi et al. (20I I) and Medeiros and Aidar (20II). Joly et al. (20I2) highlighted the increase of palm species in higher altitudes, reaching more than 400 ind.ha $^{-1}$ in the Montane Ombrophilous Dense Forest, as well as Cyatheaceae species. In the studied plot, Cyatheaceae family prevailed in the lower parts of the hillside, while the Arecaceae individuals had wider distribution throughout the plot. The distribution pattern suggest that these species could be potential competitors as they both have similar architecture, form dense populations in mountainous regions and usually share similar habitats (TRYON; TRYON, 1982).

The distribution of all live trees into diameter classes showed an exponential negative or unbalanced inverted J-shaped curve, i.e., there was a predominance of young individuals in early regeneration stages and lower values of diameter and a marked decrease in the larger classes (Figure 2). The first two classes (DHB up to $20 \mathrm{~cm}$ ) contained about $86 \%$ of the individuals sampled, followed by the classes with DHB up to $40 \mathrm{~cm}$ with $12 \%$, and the other classes with the remaining $2 \%$ of the total individuals. The average diameter for the entire sample was $12.8 \mathrm{~cm}$, with the highest value from an Alchornea triplinervia $(108.3 \mathrm{~cm})$ individual.

The individual distribution by height classes (Figure 3) showed low overall height with an average of $10.3 \mathrm{~m}$, a result similar to other studies developed in the Atlantic Forest (OGATA; GOMES, 2006; MEDEIROS; AIDAR, 20I I; JOLY et al., 20I2). Most individuals (74\%)

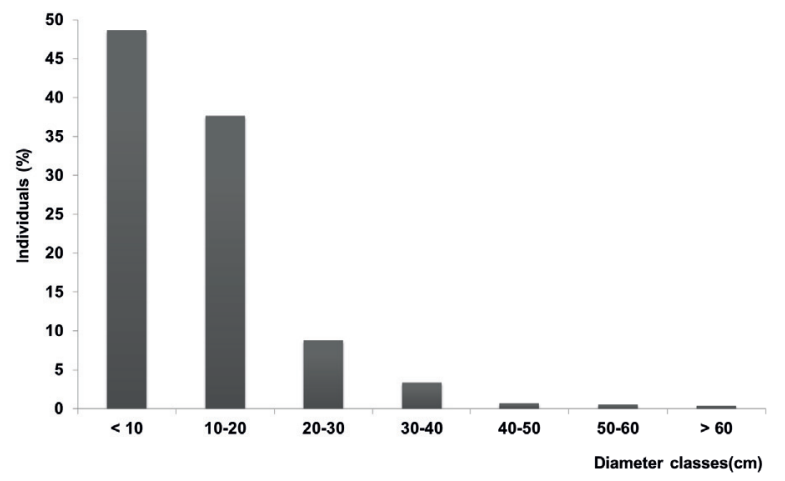

FIGURE 2 Distribution of individuals in their respective diameter classes (in centimeters) at Santa Virgínia Nucleus. The $y$-axis represents the percentage of individuals.

showed heights between 6-16 m, however, less than $5 \%$ of individuals have heights between $18-26 \mathrm{~m}$, similar to results of Joly et al. (20/2). The taller individuals are members of the Araliaceae, Chrysobalanaceae, Elaeocarpaceae, Euphorbiaceae, Fabaceae, Lauraceae, Melastomataceae, Myrtaceae and Phyllanthaceae families. They could form an emergent canopy and are, mainly, classified as early and late secondary in the succession. Moreover, representatives of the Cyatheaceae and Arecaceae ( $E$. edulis) families are typical of the understory of a forest, reaching average of 5.3 and $10.9 \mathrm{~m}$ high. The understanding of stem diameter and height represent the growth and, consequently, the forest structure and biomass (COOMES; ALLEN, 2007).

The environmental conditions vary along altitudinal gradients. At higher altitudes, the low-level clouds and fog formation could reduce the annual irradiance (SOUSA NETO et al., 20I I). This condition, associated with the reduction of the air and soil temperature and prevalence of strong winds, could be the cause of the decrease in tree heights and the

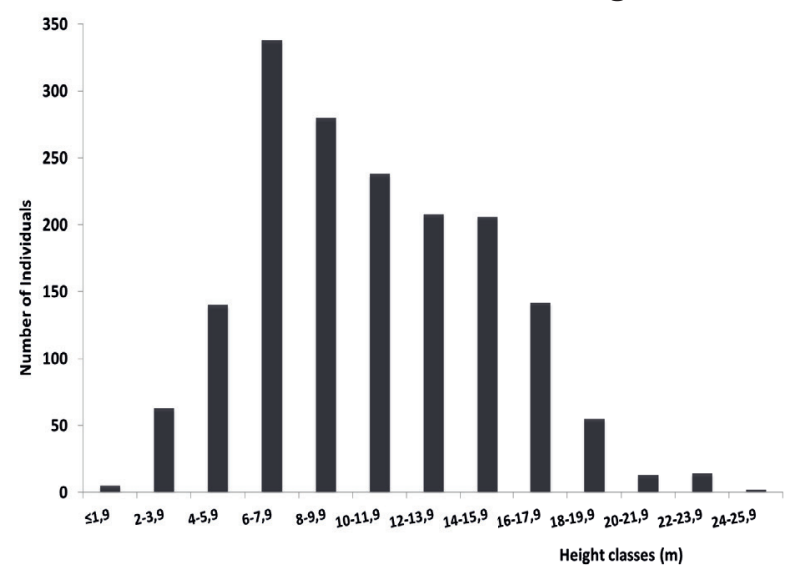

FIGURE 3 Distribution of individuals in size classes (height in meters) at Santa Virginia Nucleus. The $y$-axis represents the number of individuals. 
increase in diameter in montane forests (ALVES et al., 2010). Also, the distribution of light is more asymmetric in steeper terrain (ALVES et al., 2010), resulting in individuals shorter, with larger diameters instead of height, and wider crowns (ALVES; SANTOS, 2002).

According to the successional classification, the group "Not characterized (NC)" is composed of 20 species (I4\%), "Pioneer (PI)" by I4 species $(9.8 \%)$, "Early secondary (ES)" by 32 species (22.4\%) and "Late secondary (LS)" 77 species (54\%) (Figure 4A). Guariguata and Ostertag (200I) assumed that forests at early stages are mainly influenced by factors that guide colonization, related to germination and sprouts. In contrast to later stages, when biotic and abiotic competitive ability and tolerance of environmental conditions among species (determined primarily by rates of species-specific growth, longevity, maximum size at maturity and the degree of shade tolerance) largely dictate patterns of species replacement over time. The level of succession also contributes to nitrogen fertilization of the soil, carbon accumulation in biomass, composition of species and stem density (KOERSELMAN; MEULEMAN, 1996). Due to the evolution in succession, gaps suitable for the light demanding species tend to be rare, decreasing the number of pioneer species (DENSLOW, 1980), while Finegan (1996) pointed that the proximity to a mature forest could improve the capacity of a secondary area to regenerate, especially because of the seed rain, remnant trees, pollinators, and dispersers.

The abundance of early secondary individuals ( 853 ind.) (Figure 4B), the presence of species considered typical of disturbed environments, such as Vernonia sp.I, Alchornea triplinervia, Hyeronima alchorneoides and Casearia decandra, the land use history, presence of gaps and several bamboo clumps suggest that the studied area is secondary with more than 40 years of succession (TABARELLI; MANTOVANI, 1999). However, species diversity and abundance of individuals (575) from the late secondary category suggest that the initial phase of regeneration is progressing toward the climax condition. The diversity of species from Myrtaceae and Lauraceae families indicate a progress in the succession, although the abundance of Alchornea triplinervia has reinforced the classification in middle secondary stage (TABARELLI; MANTOVANI, 1999). This information is highly significant to a better understanding of the community's gas exchange potential around the flux tower (FREITAS, 20I2), as secondary forests show normally high productivity and biomass accumulation pattern when compared with mature forests (BROWN; LUGO, 1990).
The inverted J-shaped standard of diameter classes is common in tropical forests with a diversity of age and composition, also found in Medeiros and Aidar (20I I). It represents the age distribution of a community, since there is a direct relationship between the increase in the diameter and the age of the plant (OLIVER; LARSON, 1996). Furthermore, it indicates a self-regeneration within the community, with a predominance of recruited individuals with lower diameter values (SILVA; NASCIMENTO, 200I). The difference between the frequencies of individuals in the first and the last classes indicates that the life cycle was blocked by some past event, such as the selective cutting of larger trees (NEVES; PEIXOTO, 2008) and could reinforce the successional status of the area as middle secondary.

The calculations of the aboveground biomass using allometric models indicated that the biomass accumulated was of $166.3 \mathrm{Mg} \cdot \mathrm{ha}^{-1}$, with tree species representing the greatest amount of biomass (I55.6 $\mathrm{Mg} \cdot \mathrm{ha}^{-1}$ or $\left.93.6 \%\right)$, followed by the palm trees $(9.2$

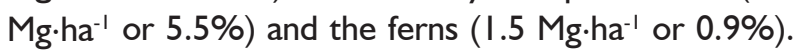

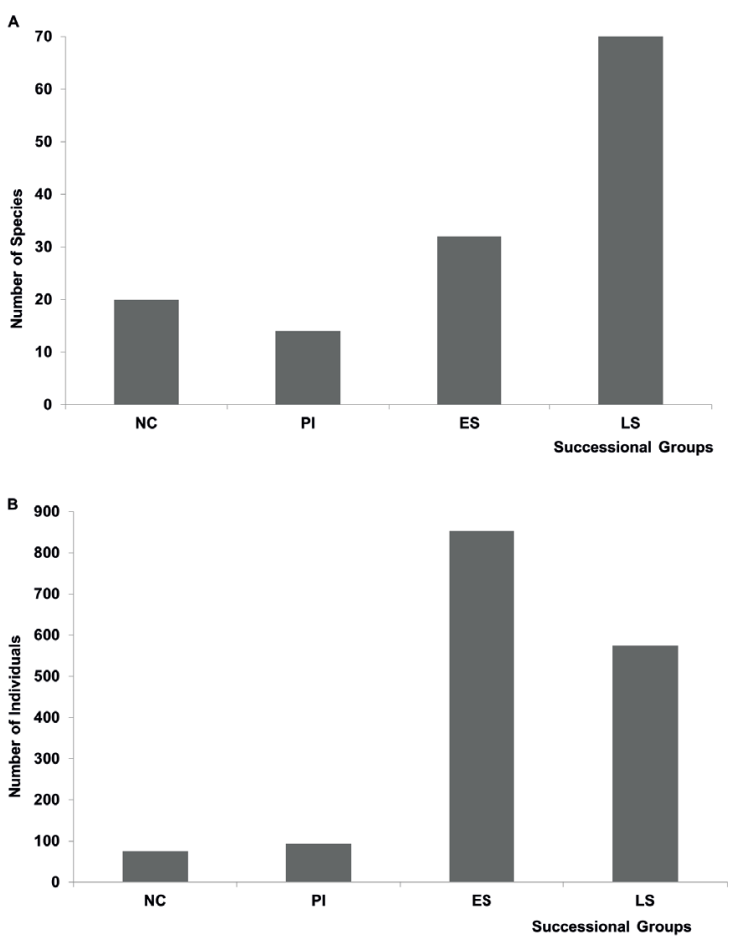

FIGURE 4 Distribution of species and individuals in their respective successional groups at Santa Virgínia Nucleus. Figure "A" represents number of species and "B" individuals. NC - not characterized, $\mathrm{PI}$ - pioneers, ES - early secondary and ST - late secondary. The $y$-axis represents the number of species (A) e individuals (B). 
The biomass at Montane Ombrophilous Dense Forest in the study of Medeiros and Aidar (20II) was similar to this study, $189.2 \mathrm{Mg} \cdot \mathrm{ha}^{-1}$. The slightly higher result may be due to extrapolation errors, since the area sampled by these authors was of 0.2 ha and the result was estimated for I ha, unlike in the present study where exactly I ha of forest was surveyed. Padgurschi (2010) and Alves et al. (20l0) obtained higher montane forest biomass results in mature forest, $282.6 \mathrm{Mg} \cdot \mathrm{ha}^{-1}$ and $27 \mathrm{l} .7 \mathrm{Mg} \cdot \mathrm{ha}^{-1}$, respectively. As we propose that the studied area is evolving from a logged to a late secondary forest, the aboveground biomass also has the tendency to increase with time, when species with higher values of wood density and the basal area would influence it (HOUGHTON et al., 2009).

Structure, composition, and function are the three essential attributes of forest ecosystems. Mandal and Joshi (2014) suggested that these characteristics could change in response to climate, topography, soil and disturbances. The aboveground live biomass varies widely in neotropical forests due to regional differences in the individual size, wood density, species composition, soil fertility, topography and disturbance (VIEIRA et al., 2004; CHAVE et al., 2005; MALHI et al., 2006; VIEIRA et al., 2008). The general trends for forests from high altitudes are the decline in stature and reduction in the aboveground biomass, while there is an increase in wood density (Aiba and Kitayama, 1999; Moser et al., 2007). This pattern is a result of the limiting factors acting in these forests, mainly affecting photosynthesis, transpiration and nutrient availability (AIBA; KITAYMA, 1999; RAICH et al., 2006). However, Alves et al. (2010) found that in the Atlantic Forest, the biomass and abundance of large individuals are incremented with the increasing altitude.

Individuals with $\mathrm{DBH} \geq 40 \mathrm{~cm}$, even if weakly represented in the sampled area (I.6\%) had influenced in the total biomass calculation - $43 \mathrm{Mg} \cdot \mathrm{ha}^{-1}$ or $26 \%$ of that value. In contrast, the diameters inferior to $10 \mathrm{~cm}$ (48.7\%) had accumulated only $6.7 \%$ or I I.I Mg.ha ${ }^{-1}$ of the total biomass (Figure 5). Considering the height, those higher than $20 \mathrm{~m}$ ( $1.7 \%$ of total) had $20 \%$ of the biomass (33.8 $\mathrm{Mg} \cdot \mathrm{ha}^{-1}$ ) and those with wood density equal or higher than $0.8 \mathrm{~g} . \mathrm{cm}^{3}$ ( $\left.1 \mathrm{l} \%\right)$ had $10.8 \%$ of the

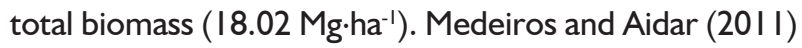
researching a plot at Montane Ombrophilous Dense Forest found that diameters of up to $13 \mathrm{~cm}$ comprised $60 \%$ of the individuals and $7.4 \%$ of the total biomass, similarly to the results found by Vieira et al. (2004) in a Central Amazon forest, where trees with diameters between 10 and $29.9 \mathrm{~cm}$ corresponded to approximately $80 \%$ of the individuals sampled, and contributed only with 26.4 to $32.9 \%$ of the total estimated biomass.

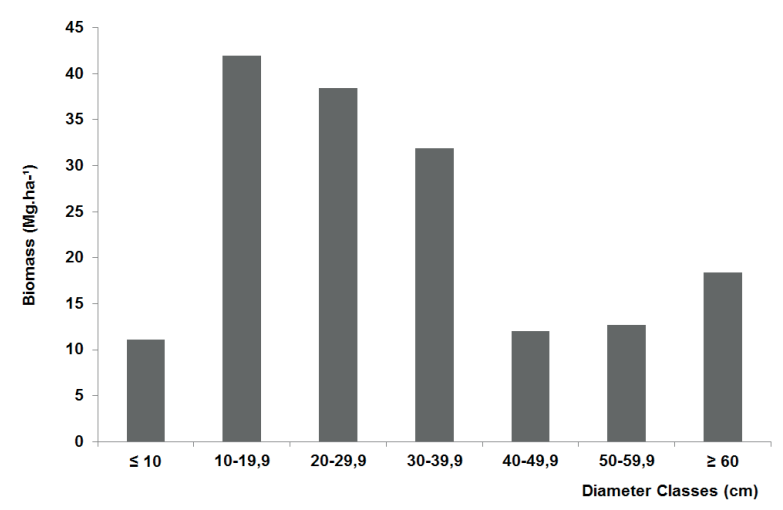

FIGURE 5 Distribution of aboveground biomass in the respective diameter classes at Santa Virgínia Nucleus. The $y$-axis represents live aboveground biomass $\left(\mathrm{Mg} \cdot \mathrm{ha}^{-1}\right)$.

\section{CONCLUSIONS}

The forest studied in a secondary stage of regeneration, after significant past impact more than 40 years ago. However, if we consider the little amount of pioneers species (and individuals) and the higher number of early and late secondary species (and individuals) we can conclude that the site is evolving to a more mature condition along succession and could be considered in middle stage. We can predict that aboveground biomass accumulation would increase in the future decades, especially due to the change in the composition (TABARELLI; MANTOVANI, 1999). The results will help a better understanding of the gas exchanges between atmosphere and biosphere in the area and will provide subsidies for the development a model of carbon balance in the Montane Ombrophilous Dense Atlantic Forest from southeastern Brazil.

\section{ACKNOWLEDGMENTS}

The authors would like to thank people involved in fieldwork - Renato Belinello, Wagner Toledo, Msc. Fernanda Cassemiro, Ms. Filipe Pikart, Ms. Janaína Silva and Msc. Giampiero Bini Cano; plant identification Dr. Maíra Padgurschi and staff of Vegetal Taxonomy Laboratory of Unicamp; and English review - Espaço da Escrita - Unicamp. This work was supported by the Coordenação de Aperfeiçoamento de Pessoal de Nível 
Superior (Capes), FAPESP Thematic projects "Carbon Tracker and Water Availability: Controls of Land Use and Climate Changes" (FAPESP 08/58I20-3), and "Gradiente Funcional: Composição florística, estrutura e funcionamento da Floresta Ombrófila Densa dos Núcleos Picinguaba e Santa Virgínia, do Parque Estadual da Serra do Mar, São Paulo, Brasil" (Processo FAPESP 03/I2595-7).

\section{REFERENCES}

AIBA, S.; KITAYAMA, K. Structure, composition and species diversity in an altitude-substrate matrix of rain forest tree communities on Mount Kinabalu, Borneo. Plant Ecology, v.140, n. 2, p.139-157, 1999.

AIDAR, M.P.M.; SCHMIDT, S.; MOSS, G, STEWART, G.R.; JOLY, C.A. Nitrogen use strategies of neotropical rainforest trees in threatened Atlantic Forest. Plant Cell and Environment, v. 26, n.3, p.389-399, 2003.

ALVES, L.F; VIEIRA, S.A.; SCARANELLO, M.A.; CAMARGO, P.B.; SANTOS, F.A.M.; JOLY, C.A.; MARTINELLI, L.A. Forest structure and live aboveground biomass variation along an elevational gradient of tropical moist forest (Brazil). Forest Ecology and Management, v. 260, n.5, p.679-69I, 2010.

ALVES, L.F.; SANTOS, F.A.M. Tree allometry and crown shape of four tree species in Atlantic rain forest, southeast Brazilian. Journal of Tropical Ecology, v. 18, n. 2, p.245260, 2002.

ANGIOSPERM PHYLOGENY GROUP/APG. An update of the Angiosperm Phylogeny Group classification for the orders and families of flowering plants: APG III. Botanical Journal of the Linnean Society, v. I6I, p. I05-I2I, 2009.

BROWN, S.; LUGO, A. E. Tropical secondary forests. Journal of Tropical. Ecology, v. 6, n.I, p.I- 32, 1990.

BRUIJNZEEL, L.A.; VENEKLAAS, E.J. Climatic conditions and Tropical Montane Forest productivity: the fog has not lifted yet. Ecology, v.79, n.I, p. 3-9, 1998.

CHAVE, J.; MULLER-LANDAU, H. C.; BAKER, T. R.; EASDALE, T. A.; TER STEEGE, H., WEBB, C. O. Regional and phylogenetic variation of wood density across 2456 neotropical tree species. Ecological Applications, v. I6, n. 6, p.2356-2367, 2006.

CHAVE, J.;ANDALO, C.; BROWN, S.; CAIRNS, M.; CHAMBERS, J.C.; EAMUS, D.; FÖLSTER, H.; FROMARD, F.; HIGUCHI, N.; KIRA, T.; LESCURE, J.; NELSON, B.W.; OGAWA, H.; PUIG, H.; RIÉRA, B.; YAMAKURA, T. Tree allometry and improved estimation of carbon stocks and balance in tropical forests. Oecologia, v. I45, n. I, p.87-99, 2005.
COOMES, D.A.; ALLEN, R.B. Effects of size, competition and altitude on tree growth. Journal of Ecology, v.95, p. I0841097, 2007.

CRIA. Centro de Referência em Informação Ambiental - speciesLink. http://splink.cria.org.br/ (Access in March/2016).

DENSLOW, J.S. Patterns of plant species diversity during succession under different disturbance regimes. Oecologia, v.46, n. I, p. I8-21, 1980.

FERSTER, C. J.; TROFYMOW, J. A.; COOPS, N. C.; CHEN, B.; BLACK, T. A. Comparison of carbon-stock changes, eddycovariance carbon fluxes and model estimates in coastal Douglas-fir stands in British Columbia. Forest Ecosystems, v.2, n.I, p.13, 2015.

FIDALGO, O.; BONONI, V.L.R. Técnicas de coleta, preservação e herborização de material botânico. Manual 4. Instituto de Botânica, São Paulo, 1984.

FINEGAN, B. Pattern and process in neotropical secondary rain forests: the first 100 years of succession. Tree, v.lI, n.3, p.I19-124, 1996.

FLORA DO BRASIL 2020. Jardim Botânico do Rio de Janeiro. Disponível em: < http://floradobrasil.jbrj.gov.br/ >. Acesso em: 30 Mai. 2016

FREITAS, H.C. A influência dos transportes advectivos na estimativa do fluxo líquido do ecossistema: um estudo de caso para a Mata Atlântica com uso de técnicas micrometeorológicas. Tese de Doutorado Escola Superior de Agricultura "Luiz de Queiroz", USP, Piracicaba. 84p., 2012.

GALINDO-LEAL, C.; CÂMARA. I.G. Atlantic forest hotspots status: an overview. In: Mata Atlântica: biodiversidade, ameaças e perspectivas. Carlos Galindo-Leal, Ibsen de Gusmão Câmara (Ed.); traduzido por Edma Reis Lamas. São Paulo: Fundação SOS Mata Atlântica — Belo Horizonte: Conservação Internacional, pp. 3-I I, 2005.

GANDOLFI, S.; LEITÃO FILHO, H.; BEZERRA, C. L. F. Levantamento Florístico e caráter sucessional das espécies arbustivo-arbóreas de uma Floresta Mesófila Semidecídua no município de Guarulhos, SP. Brazilian Journal of Botany, v.55, n.4, p.753-767, 1995.

GOMES, J.A.M.A.; BERNACCI, L.C.; JOLY, C.A. Diferenças florísticas e estruturais entre duas cotas altiduninais da Floresta Ombrófila Densa Submontana Atlântica, do Parque Estadual da Serra do Mar, município de Ubatuba/SP, Brasil. Biota Neotropica, v. I I, n.2, 201 I.

GUARIGUATA, M.R.; OSTERTAG, R. Neotropical secondary forest succession: changes in structural and functional characteristics. Forest Ecology and Management, v. I48, n. I, p. I85-206, 200I. 
HAMILTON, L. S.; JUVIK, J. O.; SCATENA, F. N. (eds.) Tropical Montane Cloud Forests. Springer, New York, 1995.

HOUGHTON, R.A.; HALL, F.; GOETZ, S.J. Importance of biomass in the global carbon cycle. Journal of Geophysical Research, v. I|4, n. G2, 2009.

HOUGHTON, R. A. Balancing the global carbon budget. Annual Review of Earth and Planetary Sciences, v.5, p.3।3-347, 2007.

HOBBS, R.; HIGGS, E.; HARRIS, J. A. Novel ecosystems: implications for conservation and restoration. Trends in Ecology and Evolution, v.24, n. I I, p.599-605, 2009.

INSTITUTO BRASILEIRO DE GEOGRAFIA E ESTATÍSTICA/ IBGE. Manuais técnicos em geociências - Manual Técnico da Vegetação Brasileira. Rio de Janeiro, 27I p. 2012.

INSTITUTO FLORESTAL. Unidades de Conservação do Estado de São Paulo. Disponível em: http://www.iflorestal. sp.gov.br/unidades_conservacao/index.asp. Acesso em I4 jan. 2010.

INSTITUTO FlORESTAL. Plano de Manejo do Parque Estadual da Serra do Mar. São Paulo, 2006.

JOLY, C.A.; METZGER, J.P.; TABARELLI, M. Experiences from the Brazilian Atlantic Forest: ecological findings and conservation initiatives. New Phytologist Tansley Review, v. 204, n.3, p. 459-473, 2014.

JOLY, C.A.; ASSIS, M.A.; BERNACCI, L.C.; TAMASHIRO, J.Y.; CAMPOS, M.C.R.; GOMES, J.A.M.A.; LACERDA, M.S.; SANTOS, F.A.M.; PEDRONI, F; PEREIRA, L.S. et al. Floristic and phytosociology in permanent plots of the Atlantic Rainforest along an altitudinal gradient in southeastern Brazil. Biota Neotropica, v. I2, n. I, p. I25-I45, 2012.

JOLY, C.A.; AIDAR, M.P.M.; KLINK, C.A.; MCGRATH, D.G.; MOREIRA, A. G.; MOUTINHO, P.; NEPSTAD, D.C.; OLIVEIRA, A. A.; POTT, A.; RODAL, M.J.N.; SAMPAIO, E.V.S.B. Evolution of the Brazilian phytogeography classification systems: implications for biodiversity conservation. Ciência e Cultura, v. 5I, n.5-6, p.33I-348, 1999.

KEELING, H.C.; PHILLIPS, O.L. The global relationship between forest productivity and biomass. Global Ecology and Biogeography, v. I6, n. 5, p.618-63।, 2007.

KLJUN, N.; ROTACH, M.W.; SCHMID, H.P. A threedimensional backward lagrangian footprint model for a wide range of boundary-layer stratifications. BoundaryLayer Meteorology, v. I03, n.2, p.205-226, 2002.

KOERSELMAN, W.; MEULEMAN, A.F.M. The vegetation N : P ratio: a new tool to detect the nature of nutrient limitation. Journal of Applied Ecology, v.33, p.|44I-|450, 1996.

KÖPPEN, W. Climatologia: con un estudio de los climas de la tierra. México: Fondo de Cultura Economica, 478p, 1948.
LIEBERMAN, D.; LIEBERMAN, M.; PERALTA, R.; HARSHORN, E.G.S. Tropical forest structure and composition on a large-scale elevational gradient in Costa Rica. Journal of Ecology, v.84, p.I37-152, 1996.

MALHI, Y.; GARDNER, T.A.; GOLDSMITH, G.R.; SILMAN, M.R.; ZELAZOWSKI, P. Tropical forests in the Anthropocene. Annual Review of Environment and Resources, v.39, p.125-159, 2014.

MALHI, Y.; WOOD, D.; BAKER, T.R.; WRIGHT, J.; PHILLIPS, O.L.; COCHRANE, T.; MEIR, P.; CHAVE, J.; ALMEIDA, S.; ARROYO, L.; HIGUCHI, N.; KILLEEN, T.J.; LAURANCE, S.G.; LAURANCE, W.F.; LEWIS, S.L.; MONTEAGUDO, A.; NEILL, D.A.; NUNEZ-VARGAS, P.; PITMAN, N.C.A.; QUESADA, C.A.; SALOMÃO, R.; SILVA, J.N.M.; LEZAMA, A.T.; TERBORGH, J.; VÁSQUEZ MARTINEZ, R. The regional variation of aboveground live biomass in oldgrowth Amazonian forests. Global Change Biology, v. I2, n. 7, p.II07-II38, 2006.

MALHI, Y.; BALDOCCHI, D.D.; JARVIS, P.G. The carbon balance of tropical, temperate and boreal forests. Plant Cell and Environment, v.22, n.6, p.7I5-740, 1999.

MANDAL, G.; JOSHI, S.P. Analysis of vegetation dynamics and phytodiversity from three dry deciduous forests of Doon Valley, Western Himalaya, India. Journal of Asia-Pacific Biodiversity, v.7, n.3, p.292-304, 2014.

MARTINS, S.C. Caracterização dos solos e serapilheira ao longo do gradiente altitudinal da Mata Atlântica, estado de São Paulo. Tese de Doutorado. Centro de Energia Nuclear na Agricultura, Universidade de São Paulo, Piracicaba, SP, 2010.

MEDEIROS, M.C.M.P.; AIDAR, M.P.M. Structural variation and content of aboveground living biomass in an area of Atlantic Forest in the State of São Paulo, Brazil. Hoehnea, v.38, n.3, p. 4|3-428, $201 \mid$.

MELILLO, J.M.; MCGUIRE, A.D.; KICKLIGHTER, D.W.; MOORE, B.; VOROSMARTY, C.J.; SCHLOSS, A.L. Global climate change and terrestrial net primary production. Nature, v.363, n.6426, p.234-238, 1993.

MOSER, G.; HERTEL, D.; LEUSCHNER, C. Altitudinal change in LAl and stand leaf biomass in tropical montane forests: a transect study in Ecuador and a pantropical meta-analysis. Ecosystems, v. I0, n. 6, p.924-935, 2007.

MUELLER-DOMBOIS, D.; ELLENBERG, H. Aims and methods of vegetation ecology. John Wiley and Sons, New York, p. 44-66, 1974.

MYERS, N.; MITTERMEIER, R.A.; MITTERMEIER, C.G.; FONSECA, G.A.B.; KENT, J. Biodiversity hotspots for conservation priorities. Nature, v. 403 , n. 6772 , p. $853-$ 858, 2000.

NASCIMENTO, H.E.M.; LAURENCE, W.F. Total aboveground biomass in central Amazonian rainforests: a landscapescale study. Forest Ecology and Management, v. I68, n. I, p. 3II-32I, 2002. 
NEVES, G.M.S.; PEIXOTO, A.L. Florística e estrutura da comunidade Arbustivo-arbórea de dois remanescentes em regeneração de floresta atlântica secundária na reserva biológica de Poço das Antas, Silva Jardim, Rio de Janeiro. São Leopoldo: Instituto Anchietano de Pesquisas. Pesquisas, botânica, v.59, p.7I-I I2, 2008.

OGATA, H.; GOMES, E.P.C. Estrutura e composição da vegetação no Parque CEMUCAM, Cotia, SP. Hoehnea, v.33, n.3, p.37I-384, 2006.

OLIVEIRA-FILHO, A.T.; FONTES, M.A.L. Patterns of floristic differentiation among Atlantic Forests in Southeastern Brazil, and the influence of climate. Biotropica, v.32, n.4b, p.793- 810, 2000.

OLIVER, C.D.; LARSON, B.C. Forest stand dynamics. John Wiley and Sons. New York, 1996.

PADGURSCHI, M.C.G.; PEREIRA, L.S.; TAMASHIRO, J.Y.; JOLY, C.A. Composição e similaridade florística entre duas áreas de Floresta Atlântica Montana, São Paulo, Brasil. Biota Neotropica, v. I I, n.2, p.00-00, 201 I.

PADGURSCHI, M.C.G. Composição e estrutura arbórea de um trecho de Floresta Ombrófila Densa Montana com taquaras na Mata Atlântica. Dissertação de Mestrado. Universidade Estadual de Campinas, Campinas, SP, 2010.

PEIXOTO, A.L. Revisão taxonômica do gênero Mollinedia Ruiz et Pavon (Monimiaceae, Monimioideae). Tese de Doutorado, Universidade Estadual de Campinas, Campinas, 1987.

PEIXOTO, A.L.; GENTRY, A. Diversidade e composição florística da mata de tabuleiro na Reserva Florestal de Linhares (Espírito Santo. Brasil). Revista Brasileira de Botânica, v.13, n.I, p.19-25, 1990.

PIELOU, E.C. Ecological diversity. John Wiley and Sons, New York, 1975.

RAICH, J.W.; RUSSELL, A.E.; KITAYAMA, K.; PARTON, W.J.; VITOUSEK, P.M. Temperature influences carbon accumulation in moist tropical forests. Ecology, v.87, $\mathrm{nl}$., p.76-87, 2006.

RIBEIRO, M.C.; METZGER, J.P.; MARTENSEN, A.C.; PONZONI, F.J.; HIROTA, M.M. The Brazilian Atlantic Forest: How much is left, and how is the remaining forest distributed? Implications for conservation. Biological Conservation, v. I42, n.6, p. I I4I-I I53, 2009.

ROCHELLE, A.L.C.; CIELO-FILHO, R.; MARTINS, F.R. Tree community structure in an Atlantic forest fragment at Serra do Mar State Park, southeastern Brazil. Biota Neotropica, v. II, n.2, p. 337-346,20II.

SCARANELLO, M.A.S.; ALVES, L.F.; VIEIRA, S.A.; CAMARGO, P.B.; JOLY, C.A.; MARTINELLI, L.A. Height-diameter relationships of tropical Atlantic moist forest trees in southeastern. Scientia Agricola, v. 69, n. I, p.26- 37, 20 I I.
SHEPHERD, G.J. FITOPAC 2.I. Campinas, 2010.

SIEGLOCH, A.E.; FROEHLICH, C.G; SPIES, M.R. Diversity of Ephemeroptera (Insecta) of the Serra da Mantiqueira and Serra do Mar, southeastern Brazil. Revista Brasileira de Entomologia, v.56, n.4, p.473-480, 2012.

SILVA, G.C.; NASCIMENTO, M.T. Fitossociologia de um remanescente de mata sobre tabuleiros no norte do estado do Rio de Janeiro (Mata do Carvão). Brazilian Journal of Botany, v.24, n.I, p.5I-62, 200 I.

SILVA, J.M.C; TABARELLI, M. Tree species impoverishment and the future flora of the Atlantic forest of northeast Brazil. Nature, v. 404, n. 6773, p. 72-74, 2000.

SOETHE, N.; LEHMANN, J.; ENGELS, C. Nutrient availability at different altitudes in a tropical montane forest in Ecuador. Journal of Tropical Ecology, v. 24, n. 04, p. 397-406, 2008.

SOSMA/INPE. Atlas dos remanescentes florestais da Mata Atlântica. Período 20I I-20I2. Relatório técnico. SOS Mata Atlântica e Instituto Nacional de Pesquisas Espaciais, 2013.

SOUSA NETO, E.; CARMO, J.B.; KELLER, M.; MARTINS, S.C.; ALVES, L.F.; VIEIRA, S.A.; PICCOLO, M.C.; CAMARGO, P.; COUTO, H.T.Z.; JOLY, C.A.; MARTINELLI, L.A. Soilatmosphere exchange of nitrous oxide, methane and carbon dioxide in a gradient of elevation in the coastal Brazilian Atlantic forest. Biogeosciences, n.8, p.733-742, 20 I I.

TABARELLI, M.; MANTOVANI, W. A regeneração de uma Floresta Tropical Montana após corte e queima (São Paulo-Brasil). Brazilian Journal of Botany, v.59, n.2, p.239-250, 1999.

TIEPOLO, G.; CALMON, M.; FERETTI, A.R. Measuring and monitoring carbon stocks at the Guaraqueçaba climate action project, Paraná, Brazil. In: K. Lin and J. Lin (eds.) International Symposium on Forest Carbon Sequestration and Monitoring. Extension Series TFRI, v. I53, p.98-I I5, 2002.

TRYON, R. M.; TRYON, A. F. Ferns and allied plants with special reference to tropical America. Springer Verlag, New York, 1982.

USDA. Soil Taxonomy: A Basic System of Soil Classification for Making and Interpreting Soil Surveys. United States Department of Agriculture, Natural Resources Conservation Service Agriculture. Handbook Number 436. 888p, 1999.

VELOSO, H.P.; RANGEL-FILHO, A.L.R.; LIMA, J.C. Classificação da vegetação brasileira adaptada a um sistema universal. IBGE. Rio de Janeiro, 199I.

VIEIRA, S.A.; ALVES, L.F; DUARTE-NETO, P..; MARTINS, S.C.; VEIGA, L.G.; SCARANELLO, M.A.; PICOLLO, M.C.; CAMARGO, P.B.; CARMO, J.B.; SOUSA NETO, E.; SANTOS, F.A.M.; JOLY, C.A.; MARTINELLI, L.A. Stocks of carbon and nitrogen and partitioning between above- and belowground pools in the Brazilian coastal Atlantic Forest elevation range. Ecology and Evolution, v. I, n.3, p.42I-434, $20 \mathrm{II}$. 
VIEIRA, S.A.;ALVES, L.F;; AIDAR, M.P.M.; ARAÚJO, L.S.; BAKER, T.; BATISTA, J.L.F; CAMPOS, M.C.; CAMARGO, P.B.; CHAVE, J.; DELITTI, W.B.C.; HIGUCHI, N.; HONORIO, E.; JOLY, C.A.; KELLER, M.; MARTINELLI, L.A.; MATTOS', E.A.; METZKER, T.; PHILLIPS, O.; SANTOS, F.A.M.; SHIMABUKURO, M.T.; SILVEIRA, M.; TRUMBORE, S.E. Estimation of biomass and carbon stocks: the case of the Atlantic Forest. Biota Neotropica, v. 8, n. 2, 2008.
VIEIRA, S.; DE CAMARGO, P.B.; SELHORST, D.; DA SILVA,R.; HUTYRA, L.; CHAMBERS, J.Q.; BROWN, I.F; HIGUCHI, N.; DOS SANTOS, J.; WOFSY, S.C.; TRUMBORE, S.E.; MARTINELLI, L.A. Forest structure and carbon dynamics in Amazonian tropical rain forest. Oecologia, v. I40, n. 3, p. 468-479, 2004. 